\title{
Multiplication of Motor-driven Microtubules for Nanotechnological Applications
}

Cordula Reuther ${ }^{1}$, Paula Santos-Otte ${ }^{1}$, Rahul Grover ${ }^{1}$, Georg Heldt ${ }^{2}$, Günther Woehlke ${ }^{3}$, Stefan Diez ${ }^{1,4,5}$

${ }^{1}$ B CUBE - Center for Molecular Bioengineering, Technische Universität Dresden, 01062 Dresden, Germany

${ }^{2}$ Fraunhofer Institute for Electronic Nano Systems, 09126 Chemnitz, Germany

${ }^{3}$ Center for Functional Protein Assemblies, Technische Universität München, 85748 Garching, Germany

${ }^{4}$ Cluster of Excellence Physics of Life, Technische Universität Dresden, 01062 Dresden, Germany

${ }^{5}$ Max Planck Institute of Molecular Cell Biology and Genetics, 01307 Dresden, Germany

\section{Supplementary movies}

Movie S1. Severing events of gliding microtubules. Rhodamine-labeled microtubules, propelled by surface-immobilized kinesin-1 motors, are severed in the presence of $100 \mathrm{nM}$ spastin. The microtubule parts, which resulted from severing, were continuously propelled and frequently severed another time. Scale bar: $10 \mu \mathrm{m}$.

Movie S2. Severing of gliding microtubules over time. Gliding, rhodamine-labeled microtubules are severed in the presence of $100 \mathrm{nM}$ spastin. Due to the severing process, the microtubule parts get shorter and thus more numerous over time, until very short pieces $(<1 \mu \mathrm{m})$ eventually detached from the surface. Scale bar: $20 \mu \mathrm{m}$.

Movie S3. Multiplication assay in a PMMA network structure. Rhodamine-labeled microtubules (magenta) were gliding for 30 minutes through a PMMA network structure while being severed by spastin and elongated with Atto-647-labeled tubulin (cyan). Multiplication solution: $10 \mu \mathrm{M}$ Taxol, $1.2 \mu \mathrm{M}$ Atto-647-labeled tubulin, $14 \mathrm{nM}$ spastin, $1 \mathrm{mM}$ GTP, $4 \mathrm{mM} \mathrm{MgCl} 2$ and $0.45 \mathrm{mg} / \mathrm{ml}$ casein. Scale bar: $20 \mu \mathrm{m}$. Assay was recorded with TIRF microscopy.

Movie S4. Representative example of a multiplying microtubule within a channel of a PMMA network structure. A rhodamine-labeled microtubule (magenta) with Atto-647labeled elongations (cyan) was gliding through the channel of a PMMA network while being severed by spastin right before a dividing channel. The two resulting microtubule parts continued to move forward and then entered different channels of the network. Multiplication solution: $10 \mu \mathrm{M}$ Taxol, 1.2 $\mu \mathrm{M}$ Atto-647-labeled tubulin, $19 \mathrm{nM}$ spastin, $1 \mathrm{mM}$ GTP, $4 \mathrm{mM} \mathrm{MgCl}$ and $0.45 \mathrm{mg} / \mathrm{ml}$ casein. Scale bar: $5 \mu \mathrm{m}$. Sped up 15x.

Movie S5. Gliding microtubules before and after multiplication in a PMMA network structure. Gliding rhodamine-labeled microtubules (magenta) corresponding to Fig. 5C and D (grey panel) before (left) and after $30 \mathrm{~min}$ multiplication (right). Atto-647-labeled elongations (cyan) are observed for the right movie. Scale bar: $20 \mu \mathrm{m}$. Sped up 15x. 


\section{Supplementary Figures}

\section{Elongation with Kip2}
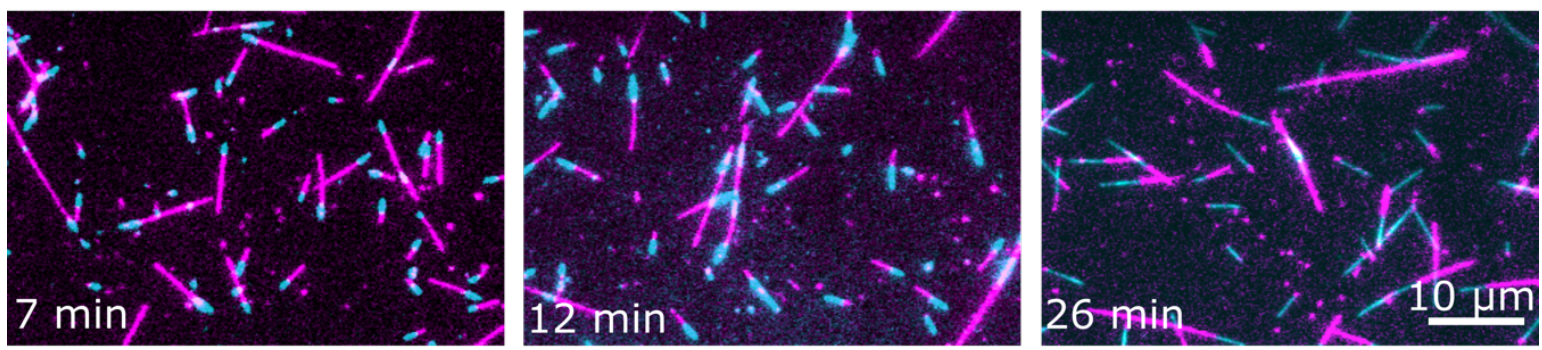

Rhodamine microtubules Atto488 elongations

Supplementary Figure 1. Elongation of gliding microtubules in the presence of kip2. Time series of color overlays showing the elongation of microtubules at $30{ }^{\circ} \mathrm{C}$. The assay was started with rhodamine-labeled microtubules (magenta) and for elongation $5 \mu \mathrm{M}$ AlexaFluor-488-labeled tubulin (cyan) was added together with 50 nM kip2 and 1 mM GTP.

A

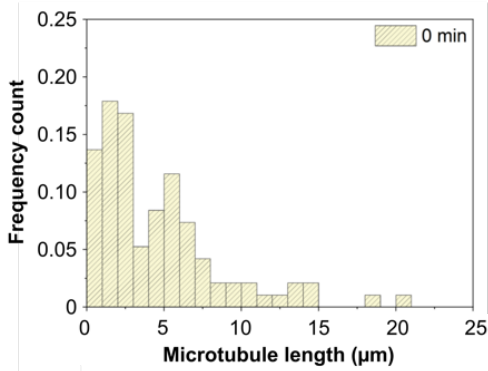

B

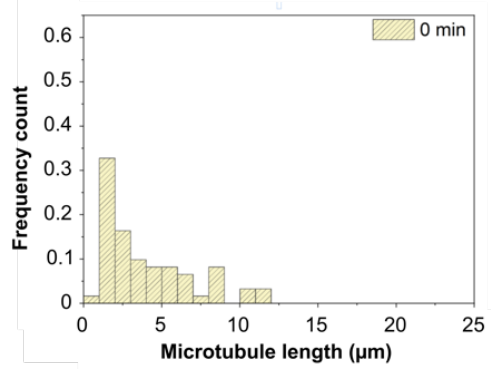

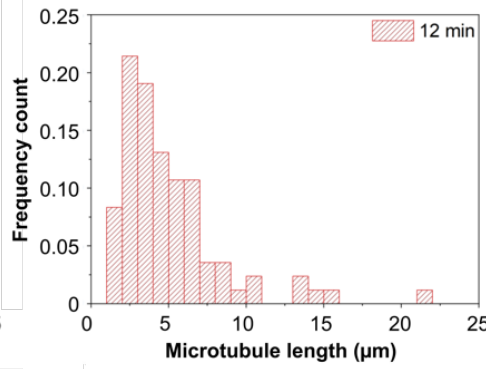
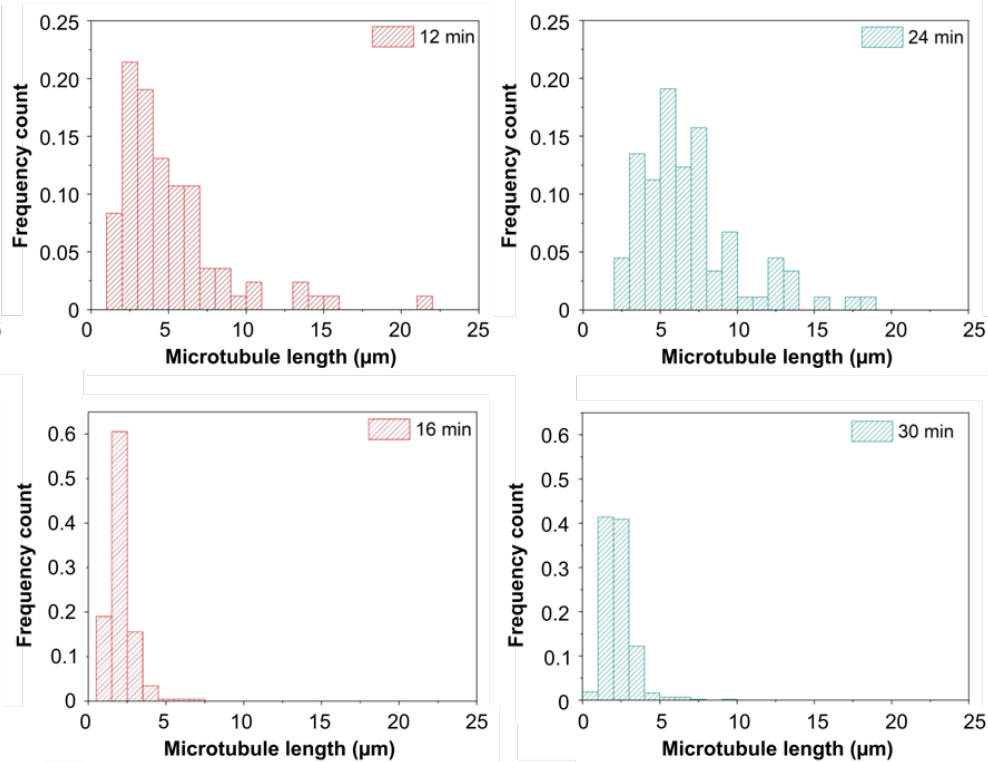

Supplementary Figure 2. Length histograms of microtubules. Microtubule lengths at different time points of $A$ ) the elongation process ( $1.2 \mu \mathrm{M}$ tubulin, $1 \mathrm{mM}$ GTP, $10 \mu \mathrm{M}$ Taxol; $\left.30^{\circ} \mathrm{C}\right)$ and $\left.\mathrm{B}\right)$ the multiplication process $(1.2 \mu \mathrm{M}$ tubulin, $1 \mathrm{mM}$ GTP, $10 \mu \mathrm{M}$ Taxol, $60 \mathrm{nM}$ spastin; $30^{\circ} \mathrm{C}$ ) in Fig. $3 \mathrm{C}$ and $4 \mathrm{C}$, respectively. 


\section{Supplementary Tables}

\begin{tabular}{|l|c|c|c|c|}
\hline & \multicolumn{4}{|c|}{ Average microtubule gliding velocity ( \pm Stdv) } \\
\hline & before the process & after $2 \mathrm{~min}$ & after $7 \mathrm{~min}$ & after $12 \mathrm{~min}$ \\
\hline Severing $\left(28^{\circ} \mathrm{C}\right)$ & $777 \pm 79 \mathrm{~nm} / \mathrm{s}$ & $723 \pm 69 \mathrm{~nm} / \mathrm{s}$ & $594 \pm 83 \mathrm{~nm} / \mathrm{s}$ & $540 \pm 76 \mathrm{~nm} / \mathrm{s}$ \\
\hline & before the process & after $5 \mathrm{~min}$ & after $15 \mathrm{~min}$ & after $25 \mathrm{~min}$ \\
\hline Elongation $\left(30^{\circ} \mathrm{C}\right)$ & $860 \pm 120 \mathrm{~nm} / \mathrm{s}$ & $834 \pm 125 \mathrm{~nm} / \mathrm{s}$ & $853 \pm 102 \mathrm{~nm} / \mathrm{s}$ & $899 \pm 85 \mathrm{~nm} / \mathrm{s}$ \\
\hline Multiplication $\left(30^{\circ} \mathrm{C}\right)$ & $928 \pm 61 \mathrm{~nm} / \mathrm{s}$ & $916 \pm 80 \mathrm{~nm} / \mathrm{s}$ & $835 \pm 116 \mathrm{~nm} / \mathrm{s}$ & $842 \pm 96 \mathrm{~nm} / \mathrm{s}$ \\
\hline
\end{tabular}

Supplementary Table 1. Average microtubule gliding velocities. The gliding velocities were evaluated before starting the respective process (severing, elongation or multiplication) and at different time points after starting it. The experimental conditions were the following: (i) Severing: $48 \mathrm{nM}$ spastin, (ii) Elongation: $1.2 \mu \mathrm{M}$ tubulin and (iii) Multiplication: $1.2 \mu \mathrm{M}$ tubulin, $72 \mathrm{nM}$ spastin.

\begin{tabular}{|c|c|c|c|c|c|}
\hline \multicolumn{3}{|c|}{ Figure 2D: Temperature dependence } & \multicolumn{3}{|c|}{ Figure 2E: ATP dependence } \\
\hline Compared $\mathrm{T}\left[{ }^{\circ} \mathrm{C}\right]$ & P-value & $\begin{array}{l}\text { Significant differences } \\
\text { at } a=0.01\end{array}$ & $\begin{array}{c}\text { Compared ATP } \\
\text { concentration [mM] }\end{array}$ & P-value & $\begin{array}{c}\text { Significant differences } \\
\text { at } a=0.01\end{array}$ \\
\hline 15 vs 18 & $4.46 \mathrm{E}-04$ & yes & 0.1 vs 0.25 & 5.77E-03 & yes \\
\hline 15 vs 21 & $4.46 \mathrm{E}-04$ & yes & 0.1 vs 0.5 & $5.66 \mathrm{E}-03$ & yes \\
\hline 15 vs 24 & 4.34E-04 & yes & 0.1 vs 1 & 5.77E-03 & yes \\
\hline 15 vs 27 & $4.13 E-04$ & yes & 0.1 vs 5 & $5.29 \mathrm{E}-03$ & yes \\
\hline 15 vs 30 & $4.28 \mathrm{E}-04$ & yes & 0.25 vs 0.5 & $4.31 \mathrm{E}-04$ & yes \\
\hline 15 vs 33 & $4.31 \mathrm{E}-04$ & yes & 0.25 vs 1 & $1.81 \mathrm{E}-04$ & yes \\
\hline 15 vs 35 & $4.28 \mathrm{E}-04$ & yes & 0.25 vs 5 & $1.43 \mathrm{E}-03$ & yes \\
\hline 18 vs 21 & $1.83 \mathrm{E}-04$ & yes & 0.5 vs 1 & 0.012 & no \\
\hline 18 vs 24 & $1.79 \mathrm{E}-04$ & yes & 0.5 vs 5 & 0.071 & no \\
\hline 18 vs 27 & $1.72 \mathrm{E}-04$ & yes & 1 vs 5 & $9.60 \mathrm{E}-04$ & yes \\
\hline 18 vs 30 & $1.77 \mathrm{E}-04$ & yes & & & \\
\hline 18 vs 33 & $1.78 \mathrm{E}-04$ & yes & & & \\
\hline 18 vs 35 & $1.77 \mathrm{E}-04$ & yes & \multirow{2}{*}{\multicolumn{3}{|c|}{ Figure 2F: Tubulin dependence }} \\
\hline 21 vs 24 & $6.39 \mathrm{E}-04$ & yes & & & \\
\hline 21 vs 27 & $1.72 \mathrm{E}-04$ & yes & \multirow{2}{*}{$\begin{array}{l}\text { Compared tubulin } \\
\text { concentration [mM] }\end{array}$} & \multirow{2}{*}{ P-value } & \multirow{2}{*}{$\begin{array}{c}\text { Significant differences } \\
\text { at } a=0.01\end{array}$} \\
\hline 21 vs 30 & $1.77 \mathrm{E}-04$ & yes & & & \\
\hline 21 vs 33 & $1.78 \mathrm{E}-04$ & yes & \multicolumn{3}{|l|}{ At $48 \mathrm{nM}$ spastin } \\
\hline 21 vs 35 & $1.77 \mathrm{E}-04$ & yes & 0 vs 1 & $6.98 \mathrm{E}-03$ & yes \\
\hline 24 vs 27 & $1.68 \mathrm{E}-04$ & yes & 0 vs 2 & $1.66 \mathrm{E}-04$ & yes \\
\hline 24 vs 30 & $1.73 E-04$ & yes & 1 vs 2 & $1.76 \mathrm{E}-04$ & yes \\
\hline 24 vs 33 & $1.73 E-04$ & yes & \multicolumn{3}{|l|}{ At $120 \mathrm{nM}$ spastin } \\
\hline 24 vs 35 & $1.73 \mathrm{E}-04$ & yes & 0 vs 1 & 7.43E-07 & yes \\
\hline 27 vs 30 & $1.66 \mathrm{E}-04$ & yes & 0 vs 2 & $2.42 \mathrm{E}-06$ & yes \\
\hline 27 vs 33 & $1.67 \mathrm{E}-04$ & yes & 1 vs 2 & $7.62 \mathrm{E}-04$ & yes \\
\hline 27 vs 35 & $1.66 \mathrm{E}-04$ & yes & \multicolumn{3}{|l|}{ At $240 \mathrm{nM}$ spastin } \\
\hline 30 vs 33 & $1.72 \mathrm{E}-04$ & yes & 0 vs 1 & $7.14 \mathrm{E}-12$ & yes \\
\hline 30 vs 35 & $1.71 \mathrm{E}-04$ & yes & 0 vs 2 & $1.91 \mathrm{E}-08$ & yes \\
\hline 33 vs 35 & 0.08 & no & 1 vs 2 & $1.31 \mathrm{E}-05$ & yes \\
\hline
\end{tabular}

Supplementary Table 2. Statistical tests for data points of the left graphs in Figure 2 D-F. To check whether the data was normally distributed we used the Shapiro-Wilk test. The Mann-Whitney test was then applied for non-normally and the two-samples t-test for normally distributed data. 


\begin{tabular}{|c|c|c|c|c|c|c|c|c|c|c|c|}
\hline & \multicolumn{3}{|c|}{ Figure 2D } & \multicolumn{3}{c|}{ Figure 2E } & \multicolumn{3}{c|}{ Figure 2F } \\
\cline { 2 - 12 } & \multicolumn{3}{|c|}{ Temperature ( $\left.{ }^{\circ} \mathrm{C}\right)$} & \multicolumn{3}{c|}{ [ATP] (mM) } & \multicolumn{3}{c|}{ [Free tubulin] $(\mu \mathrm{M})$} \\
\cline { 2 - 12 } & 18 & 24 & 30 & 35 & 0.25 & 0.5 & 1 & 5 & 0 & 1 & 2 \\
\hline $\begin{array}{c}\text { Total microtubule } \\
\text { length ( } \mu \mathrm{mm})\end{array}$ & 196 & 250 & 223 & 340 & 208 & 252 & 297 & 229 & 160 & 116 & 132 \\
\hline $\begin{array}{c}\text { Total number } \\
\text { of severing events }\end{array}$ & 73 & 104 & 101 & 94 & 193 & 255 & 363 & 210 & 185 & 181 & 124 \\
\hline
\end{tabular}

Supplementary Table 3. Total microtubule length and number of severing events for the assays shown in Figure 2 D-F. Severing of gliding microtubules was performed under various conditions. The total microtubule length was measured within one field of view before adding the severing solution. The total number of severing events was counted until all microtubules had detached from the surface.

\begin{tabular}{|cccc|}
\hline Time $[\mathrm{min}]$ & $<\mathrm{L}_{n}>[\mu \mathrm{m}]$ & $<\mathrm{L}_{\mathrm{w}}>[\mu \mathrm{m}]$ & PDI \\
\hline 0 & $3.963 \pm 0.046$ & $6.008 \pm 0.202$ & $1.516 \pm 0.055$ \\
\hline 10 & $2.134 \pm 0.007$ & $2.762 \pm 0.049$ & $1.294 \pm 0.024$ \\
\hline 20 & $2.168 \pm 0.002$ & $2.350 \pm 0.007$ & $1.081 \pm 0.004$ \\
\hline 30 & $2.302 \pm 0.002$ & $2.695 \pm 0.010$ & $1.171 \pm 0.004$ \\
\hline
\end{tabular}

Supplementary Table 4. Polydispersity index of microtubules during the multiplication process. The data corresponds to the experiment shown in Fig. $4 \mathrm{C}$. 